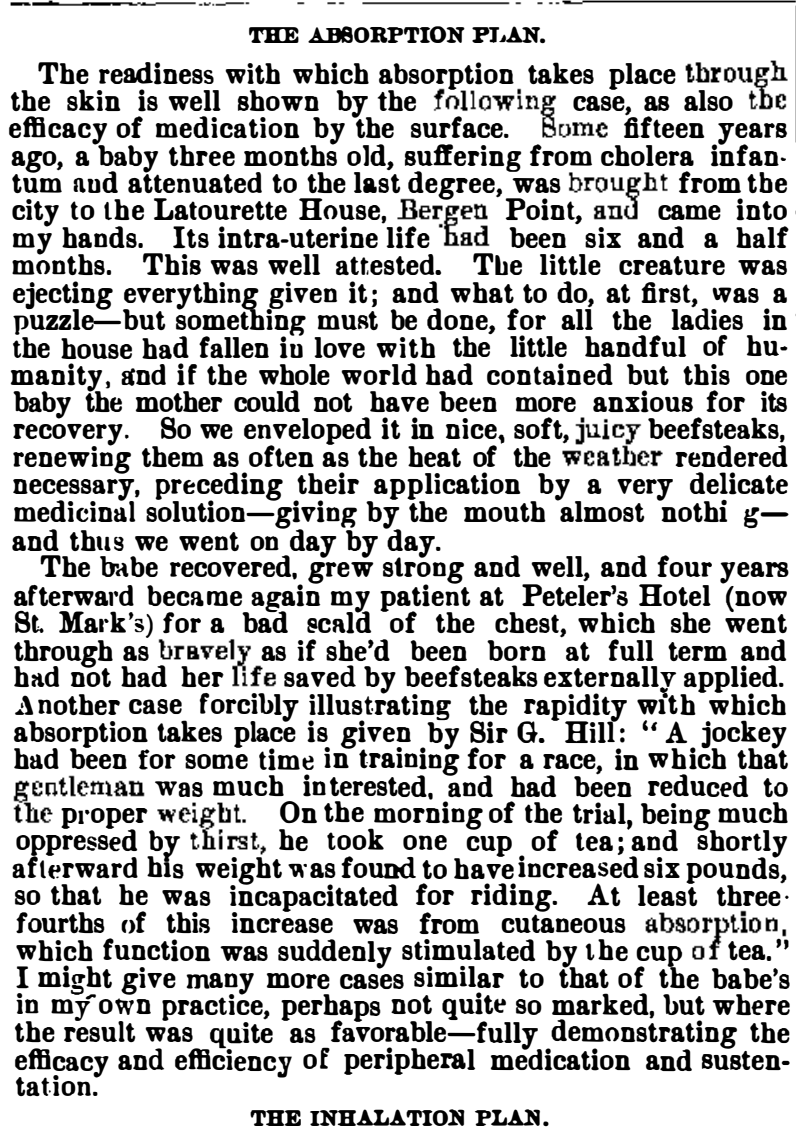

Along with this form of trestment by the surface, goes
admirably well the introduction of medicaments into the circulation by inhalation. This is a very essy a $d$ very powerful way of combating disease. As it is through the airpassages that many pernicious elements enter the system, it rectives and antidotes should be sent; and all know how efficiently agents act given this way-as chloroform, nitrous
oxide gas, oxygen, etc. In many diseases, as typhus and typhoid fever, and the
typhoid stage of other diseases, the tongue and mucous membrane become hard, rough, and as dry as a chip. The
lining of the stomach is mostly in the same condition (for the tongue is the mirror of the stomach), and medicines
placed in it are of no a vail, $f$ or there is no action, no absorption, and they lie there dead and inactive. Now, you might as well place a weasel in a tightly cemented room from of rats, as to expect medicines placed in the stomach under these circumstances to have any controlling or curative
infuence over disease; and yet this is just what is done over and over again, indeed all the time, by physicians of every
school everywhere. Not so do medicines act when given by inhalation; for, carried to the delicate vesicular membrane in thorough solution, they quickly enter the circulation, and, their neutralizing and curative work at once, and if properly and judiciously administered in conjunction with the use of face, I am sure that many diseases that are now the opprobrium of the profession will be rendered much more ame-
nable to treatment. Of course there are many affections that readily yield to treatment by the stomach. Indeed, I is liable may be controlled by the " ois medicatrix natures"
alone, and would get well without any treatmeut further han the proper regulation of diet and exercise. I do conscientiously believe that by the inhalation of me-
dicaments, carefully prepared and judiciously administered properly applied, after the skin has been well cleansed (al dead epithelium being removed), together with the use of the receptacle, a new era in the treatment of disease will be graver maladies that will surpass anything yet realized.

It has often occurred to me that the importance of at-
mospheric pressure, as affecting the heallh and general condition of mankind, is not sufficiently estimate. I believ with a celebrated writer, who said: "Give me the physical
aspect of the country, and I'll give you the character of the
inhabitants." And he might have added, the physical development also. All know that elevated situations for habi-
tations are much healthier than those in the valley or low tations are much healthier than those in the valley or low
places. Some will say because the air is purer, and in this they are correct so far as they go; but it is well known tha as we ascend, the atmospheric pressure lessens, and if we go
high enough this will be unplessantly demonstrated by
blood issuing from parts of the body. When a suitable elevation is reached, the peripheral circulation is much in-
creased; the action of the lungs is freer and fuller, and a creased; the action of the lungs is freer and fuller, and genation of the blood. Therefore, how infinitely better it summers among the mountains, than at the crowded places of fashionable resort! What a marke improvemeut there would be, not only in their physical, but mental condition
(to say nothing of morals), in the course of two or three (to say nothing of morals), in the course of two or three
years living rationally, exercising judiciously in the fine, much from asthma, spent a winter at the Fifth Avenue Hotel. After a litle be discoved lat be could live much than any lower down-and here, of course, he fixed his quarters. With the lessening of the surface pressure his correspondingly relieved. How large a number of persons suffering from lung trouble have been greatly benefited by
living in the Adirondacks! One occasionally being obliged physician of some note, who, while remaining in those mounphysician of some note, who, while remaining in those moun-
tains, enjoye fair bealth, but each time on coming to the city and remaining a short time, would have hemorrhages,
night sweats, and all the symptoms of rapid tuberculosis, rendering his fight to the mountains and remaining there

Then, again, there are some affections in which all remedies by the stomach are at times wholly inefficacious, even when that organ is in good working condition. I remember a striking case of this sort in a young lady suffering from ovarian
neuralgia, which came on regulirly every morning, and was very severe. I used everything in the way of anti-periodics, anodynes, counter-irritants, etc., that I thought applicable
to the case, but with no apparent permanent good result.
The father, tired of seeing his child suffering so, took her to The father, tired of seing his child suffering so, took her to a famous homeopath in the city, and he encouraged them
greally by saying that the trouble was not ovarian but in the greatly by saying that the trouble was not ovarian but in the
kidney, and that he'd make short work of it; but he was longer at the case than I was, and no more successful-when
the electro-chemical baths were suggested, the first one of which brought entire relief. I had at one of the hotels a
somewhat similar and severe case, in the management of which were associated with me three of the best physicians
from the city, and in this case, too, medicines had only a very slight temporary effect. The cure was effected quickly
and permanently on the ninth day by electricity. While I
think this treatment applicable in most cases, it is more particularly so in those of internal congestions and infiam-
mations, in eruptive and congestive fevers, and impaired mations, in eruptive and congestive fevers, and
circulation.

Doubtless the importance and benefit of keeping the skin will thrive and do well unless this be the case. For many years I have been a strong advocate for the
clipping of horses, and invariably have it done erery winter on every horse whose hair is too long and thick
to admit freely of the brush and curry-comb reaching the to admit freely of the brush and curry-comb reaching the
skin. Some years ago I had a horse who threw out early
in the fall a very thick, heary coat. Soon the legs began in the fall a very thick, heavy coat. Soon the legs bega
to swell, which increased daily, notwithstanding the giving of purgatives, diuretics, and free rubbing, all of which
were resorted to, as I did not want to clip so early; but all of no avail. The horse was clipped. A greasy scum
all over his skin (which the comb and brush could not reach) was removed by a wash of whisky, water, and borax; and now, after a drive all swelling was gone, and
did not return-and in eating, driving, and feeding he was Clipping the horse when the hair is coarse, thick, and lon is the very best thing that can be done, for he will do
third more work on a third less fee, and is a merciful, and not a cruel procedure. I also recommend the clipping of
cattle that are being fatted, where the hair is long and coarse, believing-indeed, knowing-that such cattle would In a word, I have long been impressed with the belief that not a tithe of the attention to the condition of the skin in dis ease, and what may be effected by medication and sustenta.
tion thereby, is given by physicians, and until this is done, the success that is given by physicians, and until this is done, the
Truined will not be reached. True, Europe has its mud-baths, and Pbiladelphia its mud and all kinds of baths, and all are good as far as they go; but to our mind, repeated daily thorough cleansing of the
skin by medicated washes, and the insuring a free
peripheral capillary circulation, are the prime essentials in peripheral capillary circulation, are the prime essentials in
the treatment of disease. - Medical Record.

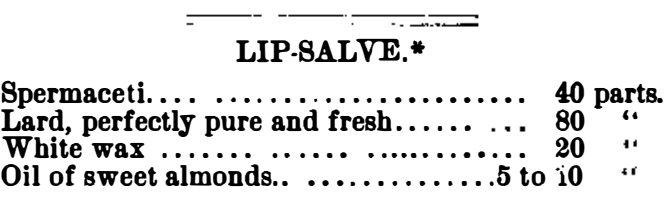

ccording to the season of the year, are melted together, the mixture colored with a sufficient quantity of glkanet, by ably perfumed, for instance, with

Oil bergamot
Oil orange

2 parts.

The mass is then poured out into moulds. It is customary

o pour it into tin-tubes, from which it is removed whe Cold Cream (Crême Celeste)

Whermaceti 30 parts,

Oil of sweet almonds.. ................ 168 .

are melted together at a gentle heat, the melted mass poured be a warm porcelain or Wedgwood mortar, stirred
be to solidify, and then intimately mixed with

Rosewater........................ 70 parts.

After stirring until cold, there may be added, for every 10 Oil of rose.

Oil of bitter almond 2 drops.

This cream is white. The following formula yield

2. White wax. ...

Olive oil, finest

166 parts.

Oil of bergamot......
Oil of bitter almond

500
100

To be prepared as the precedin

Glycerine Oream (Crême de Glycerine)

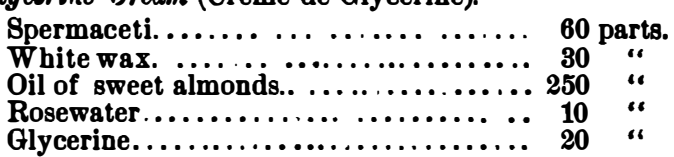

To be prepared like cold cream, and to be perfumed with

Lard, perfectly pure and fresh......... 220 parts.

Solution of potassa, containing 26 per

cent. of caustic potash.

of bitter almonds.

Triturate, in a porcelain or We gwood mortar, the lard add the alcohol and sufficient oil of bitter almonds to give it the proper flavor. Finally triturate
form, and resembles mother-of-pearl.

This cream has a handsome look, but is not so bland a the first mentioned varieties.

\section{A CATARRH CURE}

By HANB M. WIIDER.

WHEN people get a cold on the chest, as they call it, Wong other good things are prescribed the wearing of

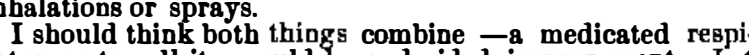
rator, so to call it-would be a decided improvement. Let the respirator be souked or sprinkled with the same balsamic
mixture which patients are ordered to use indoors as inlıalaion. When they use the respirator, prepared as above, charged with the vapors of the inhulation, although in a less egree, and in this way their air passages will be continuI got this idea from personal experience. Having a heavy cold in the head, I got a bottle of Hager's olfactorium anti

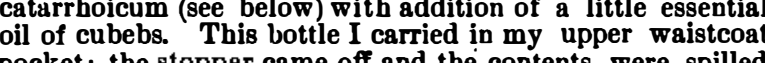
pocket; the stopper came off and the contents were spilled on my clothes. Weraring the same clothes, I was constantly of my cold and accompanying hoarseness in four days; orof weeks.

Olfactorium anticatarrhsicum.

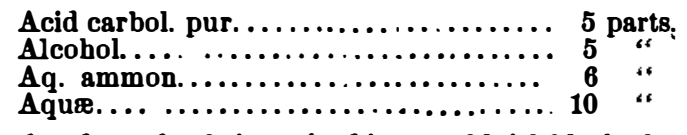

A few days after being mixed it turns bluish black (throug formation of aniline) and acquires a more disagreeable odor; lcohol by itself in one bottle, and the diluted ammonia in bottle. When using, pour from each bottle a few vapor through the nose and mouth; it is smarting at first, but one soon gets used to it. The quantity of the ammonia may, of course, be decreased.-Nero Remedies.

\section{ANTISEPTIC DRESSING OF WOUNDS.}

By MM. Gosseln and Beraeron.

TrE authors ascribe the beneficia action of these dressings not merely to the destruct
coagulation of the albumen.

POISONOUS PROPERTIES OF PHENOL. By Dr. BinnendiJK.

Tris acid acts in a direct manner upon the cerebro-spinal nervous system, and especially upon the respiratory center,
and is not without influence upon the blood. It is partly transformed in the organism, yielding hydroquinon, pyro-
catechin, etc. A portion of the phenol seems to be oxidized.

\section{VANILLIN.}

\section{HALRMANN and RERMRE.} Ax ethereal extract of vanillin, whether obtained from
vanilla pods or the oxidation product of coniferin, if shaken up with a solution of sodiumn bisulphite, yields up its vaaldenges. The compound of then destroyed by the addition of sulphuric acid, and the
pure vanillin is withdrawn from the liquid by agitation with ether. In this manner the actual vanillin, either in the natural pods or in artificial samples, may be accurately de.
termined. The authors maintain that their artificial vanillin is chemically and physically identical with the natural
product. prodiche

\section{COMPARATIVE RESEARCHES ON PTYALIN AND} By Th. Defreane.

THE action of the sa iva*is arrested by pure gastric juice; but, on the other hand, if we operate with mixed gastric
juice, containing merely or anic acids, the saccharification of

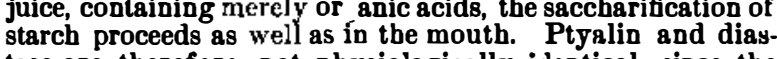
tase are, therefore, not physiologically identical, since the by pure gastric juice or by hydrochloric solutions, and even after contact with mixed gastric juice, though it still dis-

\section{- . . - - -}

THE METEOROGRAPH. W ll you, in the interest of the true history of scientific
invention, refer to the files of the BcIENTIFIC AMERICAN for May, 1854, and see if you did not publish an account of the wind that is found in the BciserTrIC AMERICAN SUPPLEMENT for January 3,1880 , and credited to Dr. Draper?
A fuller account of a paper read before the American SciA fuller account of a paper read before the American Sci-
entific Association, at eighth meeting in Washington, 1854 , may be found orpage 224 of published proceedings of that
meeting; also, there is a notice of the register in "Avnual
of Scientific Discovery" for 1855, page 192 . As the writer of this devised and constructed this part of Dr. Draper's ingenious set of automatic instruments more
than a guarter of a century ago, and made it public by dethan a quarter of a century ago, and made it public by deScientific Association, be feels himself entitled to whatever
credit is due as its originator, and fortunately his claim is easily established, unless sone one can show its existence prior to April, 1854.

As several self-registering instruments were combined, used to designate the set of automatic registers. Can any one find the word meteorograph used prior to May, 1854? N. B. WeBster.

Webster Military Institute, Norfolk, V

[Note. We are glad to be able to place the claims of we believe, in presenting before our readers. Dr. Draper, we believe, in presenting the drawings of his instruments,
made no claim to the original authorship of this class of
devicesl. 